\title{
Diseño y Validación de la Escala de Mitos del Amor Romántico
}

\author{
Design and Validation of the Scale of Myths of Romantic Love
}

\author{
Enrique Bonilla-Algovia ${ }^{1}$ y Esther Rivas-Rivero ${ }^{2}$
}

\begin{abstract}
Resumen
El objetivo de la presente investigación fue diseñar y validar la Escala de Mitos del Amor Romántico (SMRL) en una muestra de 1168 estudiantes universitarios de la Comunidad de Madrid (81.25\% mujeres y $18.75 \%$ hombres), con una edad media de 20.97 años ( $\mathrm{SD}=4.46)$. La estructura interna fue evaluada mediante varios análisis factoriales exploratorios y confirmatorios, en los que se pusieron a prueba cuatro modelos: modelo de un factor, modelo de dos factores independientes, modelo de dos factores correlacionados y modelo bifactor. Los resultados brindaron evidencias a favor de la unidimensionalidad de la SMRL y ofrecieron coeficientes de confiabilidad adecuados. Las correlaciones con los mitos sobre el amor y el sexismo ambivalente confirmaron la validez convergente. Por otra parte, a partir del modelo bifactor, se obtuvo una versión breve de la escala. En conclusión, el trabajo ofrece una herramienta con buenas propiedades psicométricas que permite analizar los mitos del amor romántico en el contexto español.
\end{abstract}

Palabras clave: amor romántico, mitos románticos, relaciones íntimas, sexismo ambivalente, validación

\begin{abstract}
The main aim of this study was to design and validate the Scale of Myths of Romantic Love (SMRL) in a sample of 1168 university students (81.25\% women and $18.75 \%$ men) from the Community of Madrid. Their mean age was 20.97 years old $(\mathrm{SD}=4.46)$. The internal structure was analyzed with exploratory and confirmatory factor analyses, in which four models were tested: one-factor model, two-independent factor model, two-correlated factor model, and bifactor model. The results provided evidence in favor of the unidimensionality of the SMRL and offered adequate reliability coefficients. Correlations with myths toward love and sexism confirmed the convergent validity. Using the results of the bifactor model, a short version of the scale was obtained. In conclusion, this research offers an instrument with good psychometric properties that allows analyzing the romantic love myths in the Spanish context.
\end{abstract}

Keywords: romantic love, romantic myths, intimate relationship, ambivalent sexism, validation

\footnotetext{
${ }^{1}$ Máster en Psicopedagogía. Personal investigador del Área de Psicología Social de la Universidad de Alcalá (FPU-UAH) y miembro de la Cátedra de Investigación Isabel Muñoz Caravaca de la UAH. Calle San Cirilo s/n, 28801, Alcalá de Henares, Madrid, España. Tel.: 918852400. Correo: enrique.bonilla@uah.es (autor de correspondencia).

${ }^{2}$ Doctora por la Universidad de Alcalá. Profesora asociada del Área de Psicología Evolutiva y de la Educación de la Universidad de Alcalá e investigadora de la Cátedra de Investigación Isabel Muñoz Caravaca de la UAH. Calle San Cirilo s/n, 28801, Alcalá de Henares, Madrid, España. Tel.: 918852400. Correo: esther.rivas@uah.es

Revista Iberoamericana de Diagnóstico y Evaluación - e Avaliação Psicológica. RIDEP · No57 · Vol.4 · 119-136 · 2020

ISSN: 1135-3848 print /2183-6051online
} 


\section{Introducción}

El amor, que ha sido objeto de estudio de diferentes disciplinas desde la segunda mitad del siglo XX (Bonilla-Algovia \& Rivas-Rivero, en prensa), ha cambiado en su concepción y desarrollo a lo largo de los años (Rocha, Avendaño, Barrios, \& Polo, 2017). Desde una perspectiva culturalista, el amor es entendido como un constructo social cuyo significado e implicaciones han ido transformándose en función del contexto histórico, el sistema social y económico y la cultura predominante (Bonilla \& Rivas, 2018); es decir, se trata de un fenómeno multidimensional situado social e históricamente que, además de aspectos emocionales, incorpora aspectos ideológicos y socioculturales (García \& Montenegro, 2014). En este sentido, lejos de ser neutro y ahistórico, se trata de un constructo cultural que depende, en buena parte, de las estructuras sociales, las normas culturales, los procesos de socialización diferencial y los mandatos de género tradicionales (BonillaAlgovia \& Rivas-Rivero, 2018; Bosch, Herrezuelo, \& Ferrer, 2019; Ferrer \& Bosch, 2013; Ferrer, Bosch, Navarro, Ramis, \& García, 2008; García, Hernández, \& Monter, 2019; Lagarde, 2001).

La literatura académica y los estudios en materia de género exponen que el ideario romántico y el modelo de amor romántico están relacionados con la producción y reproducción del orden social patriarcal y las desigualdades sociales entre hombres y mujeres (Bonilla-Algovia \& Rivas-Rivero, en prensa; Bosch et al., 2008; Caro \& Monreal, 2017; Cubells \& Calsamiglia, 2015; Esteban \& Távora, 2008; García et al., 2019; Herrera, 2014; Lagarde, 2001). El amor romántico es un fenómeno fuertemente generizado que configura prácticas relacionales desiguales (Bonilla-Algovia \& Rivas-Rivero, 2018; Bonilla \& Rivas, 2018; Bosch et al., 2019; Sánchez-Sicilia \& Cubells, 2019; Ferrer \& Bosch, 2013; Bosch et al., 2008), por lo que su significación cultural e ideológica podría ser, más si cabe en tiempos de igualdad formal, un mecanismo simbólico de reproducción de las relaciones de poder históricamente construidas en las sociedades patriarcales. El estudio de este fenómeno, entonces, debe atender a las condiciones sociohistóricas, culturales e ideológicas que lo configuran.

La fuerza simbólica es una forma de poder que, de manera invisible y prolongada, crea las condiciones necesarias para que se acepte y reproduzca el orden social (Bourdieu, 1990, 2010; Connolly \& Healy, 2004; Montesanti \& Thurston, 2015). Las estructuras de dominación como el patriarcado la utilizan con el fin de naturalizar y hegemonizar las relaciones de poder que son producto de la propia dominación (Flores \& Browne, 2017; Lozano-Verduzco, RomeroMendoza, \& Marín-Navarrete, 2016). Lo que en la actualidad se conoce como ideario del amor romántico es una ideología cultural, propia de la sociedad occidental, que prioriza un modelo concreto de relación íntima (heterosexual, monógama, estable, con papeles diferenciados y jerarquizados entre hombres y mujeres, etc.) y genera las condiciones simbólicas y prácticas que lo refuerzan (mitificación e idealización del amor romántico, necesidad del otro, abnegación, dependencia, complementariedad de género, etc.). Es decir, en tanto construcción cultural que se asimila mediante la socialización, configuraría tanto las prácticas sociales como las prácticas individuales e interpersonales en las que se sustentan las relaciones de género (Blanco, 2014) y fomentaría el rechazo de los modelos amorosos que se alejen de los dictados patriarcales. Por lo tanto, el amor romántico engloba un conjunto de representaciones, entre las que están los mitos románticos, que orientan los pensamientos, los sentimientos, las interpretaciones y los comportamientos relativos al amor y su funcionamiento (Cubells \& Calsamiglia, 2015; Sánchez-Sicilia \& Cubells, 2019).

Los mitos románticos tienen un origen diverso (Bonilla-Algovia \& Rivas-Rivero, 2019; Ferrer \& Bosch, 2013) y pueden definirse como el conjunto de creencias compartidas socialmente acerca de la naturaleza del amor (Yela, 2003); es decir, los mitos románticos vigentes en la actualidad "son el resultado de la confluencia entre el legado normativo de las tradiciones culturales y las condiciones socio-político-económicas del momento" (Yela, 2003, p. 265). En este sentido, además de representar el imaginario de la cultura occidental en torno al amor (Bonilla-Algovia \& Rivas-Rivero, 2018; Rodríguez, Lameiras, \& 
Carrera, 2015), lo configuran y ejercen presión para que este sea interiorizado. En otras palabras, los mitos románticos son creencias estructuradas y estructurantes sobre el significado del amor que están contextualizadas en un periodo sociohistórico determinado y que se concretan en prácticas y modelos relacionales específicos.

A continuación se recogen algunos de los mitos del amor romántico más comúnmente aceptados (Barrón, Martínez-Íñigo, De Paúl, \& Yela, 1999; Bosch et al., 2008; Ferrer, Bosch, \& Navarro, 2010; Yela, 2000, 2003): mito de la media naranja (creencia de que hay alguien predestinado para cada persona y que su elección como pareja es la mejor posible); mito de la pasión eterna (creencia de que la pasión inicial nunca termina y, si en algún momento lo hace, es que ya no se ama a la pareja); mito de la omnipotencia (creencia de que el amor puede con todo $\mathrm{y}$ es suficiente para solucionar los problemas); mito del matrimonio (creencia de que el amor debe conducir a la unión estable o la convivencia mediante el matrimonio); mito de los celos (creencia de que los celos son una prueba o una demostración de amor); mito de la exclusividad (creencia de que no se puede amar a más de una persona al mismo tiempo); mito del emparejamiento o de la pareja (creencia de que la monogamia heterosexual es una institución amorosa natural y universal que ha estado presente en todos los contextos históricos y culturales). Este último mito, a su vez, incluye la creencia de que no se puede ser completamente feliz sin tener una relación de pareja (Bosch et al., 2008; Ferrer et al., 2010). Otro mito que cabe mencionar es el mito de la ambivalencia o de la compatibilidad amor-violencia, que consiste en la creencia de que el amor y el maltrato son compatibles en una relación de pareja (Bosch et al., 2008; Ferrer et al., 2010). Por último, en esta investigación se ha añadido el mito de la abnegación, esto es, la creencia de que el amor implica hacer sacrificios y priorizar el bienestar de la pareja, lo que puede suponer que una persona se entregue incondicionalmente o exija a su pareja que lo haga.

Otro de los elementos que influyen en el mantenimiento de las desigualdades de género en las relaciones de pareja heterosexuales es el sexismo (Cross \& Overall, 2018; Overall \&
Hammond, 2018), que se refiere a las actitudes que se dirigen hacia las personas en función del sexo (Expósito, Moya, \& Glick, 1998; Rodríguez, Lameiras, \& Carrera, 2009a). Engloba el conjunto de creencias sobre los papeles y los comportamientos que se consideran adecuados para hombres y mujeres y establece cómo tienen que ser las relaciones entre ambos (MarquesFagundes, Megías, García-García, \& Petkanopoulou, 2015). En las últimas décadas, debido al avance de la sociedad en materia de igualdad, el sexismo tradicional ha evolucionado hacia formas de sexismo encubiertas, entre las que destaca el sexismo ambivalente (Glick \& Fiske, 1996). Este es una construcción multidimensional que incluye dos tipos de actitudes: hostiles y benévolas. Ambas dimensiones giran en torno a tres componentes compartidos: paternalismo, diferenciación de género y heterosexualidad. La principal característica del sexismo ambivalente es la coexistencia de actitudes con tonos afectivos opuestos (negativos y positivos) que, sin embargo, son igualmente discriminatorias porque ofrecen una visión de las mujeres limitada a los roles tradicionales de género (Glick \& Fiske, 1996). Por lo tanto, el respaldo de estas actitudes, bien sean hostiles o benévolas, refuerza la desigualdad en las relaciones íntimas (Glick, Sakall1-Uğurlu, Akbaş, Metin, \& Ceylan, 2016; Lee, Fiske, Glick, \& Chen, 2010; Overall \& Hammond, 2018).

Las investigaciones nacionales e internacionales coinciden en que existe una vinculación entre los mitos del amor romántico y el sexismo ambivalente (Bonilla \& Rivas, 2018; Carrascosa, Cava, Buelga, \& De Jesús, 2019; Nava-Reyes, Rojas-Solís, Greathouse, \& Morales, 2018; Rodríguez-Castro, Lameiras-Fernández, Carrera-Fernández, \& Vallejo-Medina, 2013). Las actitudes hostiles y benévolas actúan como un sistema de castigos y recompensas que mantiene las relaciones de poder entre hombres y mujeres (Glick \& Fiske, 1996). El sexismo benévolo aplica una recompensa e idealiza a las mujeres que aceptan el rol de género femenino y el sexismo hostil aplica un castigo a las mujeres que lo rechazan (Glick et al., 2016; Rodríguez, Lameiras, Carrera, \& Faílde, 2009b). En este sentido, los resultados de diversos estudios realizados en España muestran que el sexismo benévolo está más relacionado con una visión del 
amor idealizada y que el sexismo hostil, en cambio, está más relacionado con una visión del amor distorsionada y vinculada al maltrato (Rodríguez-Castro et al., 2013; Rodríguez et al., 2015). Ambos constructos, mitos románticos y sexismo ambivalente, colaborarían en el mantenimiento de las desigualdades entre hombres y mujeres y reforzarían, incluso, las creencias justificadoras de la violencia de género (Bonilla-Algovia \& Rivas-Rivero, 2019; Carbonell \& Mestre, 2018).

Barrón et al. (1999) realizaron uno de los primeros estudios sobre la aceptación de los mitos del amor romántico en España. Participaron 1949 personas de la población general con edades comprendidas entre los 18 y los 65 años. Los mitos que formaron parte del estudio fueron los siguientes: mito de la equivalencia, mito de la media naranja, mito de la exclusividad, mito de la pasión eterna, mito de la omnipotencia, mito de la fidelidad, mito del matrimonio y mito de la pareja. Cada uno de ellos fue evaluado con un ítem específico que consistía en su descripción. El grado de acuerdo se midió con una escala de 5 puntos (1=totalmente en desacuerdo; $2=e n$ desacuerdo; 3=neutral o medio; 4=de acuerdo; $5=$ totalmente de acuerdo). El estudio, si bien analizó la aceptación de las puntuaciones individuales y globales en los mitos, no tuvo como objetivo analizar la estructura interna de la escala ni las propiedades psicométricas de los ítems (Barrón et al., 1999). Posteriormente, como parte de un proyecto sobre la relación entre los mitos románticos y la violencia de género, fue diseñada la Escala de Mitos sobre el Amor (Bosch et al., 2008). El estudio contó con una muestra de 1351 personas de la población general española. En un primer momento, se elaboraron 10 ítems que evaluaban siete mitos. Los ítems consistían en una descripción de los mitos o en una frase hecha relativa a los mismos. El formato de respuesta era tipo Likert de 5 puntos, donde 1 indicaba completamente en desacuerdo y 5 , completamente de acuerdo. Los resultados reportaron que la fiabilidad de la escala era baja $(\alpha=.41)$, por lo que 2 ítems fueron eliminados. Los 8 ítems restantes evaluaban los mitos de la media naranja, de la pasión eterna, del matrimonio, de los celos, de la omnipotencia y de la ambivalencia. Un análisis factorial exploratorio mostró que los ítems se agrupaban en dos factores: Idealización del amor (6 ítems) y Vinculación amor-maltrato (2 ítems). No obstante, a pesar de la eliminación de los dos ítems mencionados, la fiabilidad global de la escala continuó siendo baja $(\alpha=.51)$ (Bosch et al., 2008). La Escala de Mitos sobre el Amor fue puesta a prueba nuevamente en el contexto español, esta vez en una muestra de población adolescente (Rodríguez-Castro et al., 2013). Un análisis factorial confirmatorio mostró un buen ajuste para un modelo reducido de 7 ítems. La estructura factorial, en línea con lo propuesto en la versión original (Bosch et al., 2008), quedo formada por dos dimensiones: Idealización del amor (5 ítems) y Vinculación amor-maltrato (2 ítems). Las propiedades psicométricas y la fiabilidad de la escala fueron adecuadas; sin embargo, al quedar reducida a 7 ítems, únicamente mide cinco mitos románticos: mito de la media naranja, mito de la pasión eterna, mito de la omnipotencia, mito de los celos y mito de la ambivalencia. Por lo tanto, partiendo de la teoría sobre los mitos románticos (Barrón et al., 1999; Bosch et al., 2008; Ferrer et al., 2010; Yela, 2000, 2003) y tomando en consideración los instrumentos previos en castellano (Bosch et al., 2008; Rodríguez-Castro et al., 2013), el objetivo de la presente investigación ha sido diseñar y validar una nueva escala que incluya el conjunto de mitos descritos anteriormente y estudiar las relaciones con el sexismo ambivalente.

\section{Método}

\section{Participantes}

La muestra está formada por 1168 estudiantes universitarios $(81.25 \%$ mujeres y $18.75 \%$ hombres) de la Comunidad de Madrid, con una edad media de 20.97 años ( $\mathrm{SD}=4.46)$. El muestreo fue intencional $y$ no aleatorio entre quienes cursaban titulaciones en Ciencias de la Educación en distintas universidades públicas de la región: Universidad Complutense de Madrid, Universidad de Alcalá, Universidad Rey Juan Carlos y Universidad Autónoma de Madrid. La Tabla 1 recoge las características sociodemográficas y de contexto. 
Tabla 1. Características de la muestra

\begin{tabular}{lccc}
\hline & $\mathrm{n}$ & $\%$ & $\begin{array}{c}\text { Media } \\
\text { (SD) }\end{array}$ \\
\hline Hombres & 219 & 18.75 & \\
Mujeres & 949 & 81.25 & \\
Edad media & & & $\begin{array}{c}20.97 \\
(4.46)\end{array}$ \\
$\begin{array}{l}\text { Situación sentimental } \\
\quad \text { Con pareja }\end{array}$ & & & \\
$\quad \begin{array}{l}\text { Sin pareja } \\
\text { Edad de la primera relación }\end{array}$ & 545 & 46.66 & \\
$\begin{array}{l}\text { intima } \\
\text { Situación económica de la } \\
\text { familia }\end{array}$ & & & 15.64 \\
$\quad \begin{array}{l}\text { Trabaja uno de los } \\
\text { progenitores }\end{array}$ & & & $(2.18)$ \\
$\quad \begin{array}{l}\text { Trabajan ambos } \\
\text { progenitores }\end{array}$ & 328 & 28.32 & \\
$\quad \begin{array}{l}\text { Trabajan otros familiares } \\
\quad \text { No trabaja nadie en la } \\
\text { familia }\end{array}$ & 38 & 3.28 & \\
\hline & 50 & 4.32 & \\
\hline
\end{tabular}

\section{Instrumento}

1. Datos sociodemográficos. Se incluyó un breve cuestionario de carácter sociodemográfico (ad hoc), en el que se preguntaba sobre la edad, el sexo, la situación socioeconómica y la situación sentimental de la persona.

\section{Escala de Mitos del Amor Romántico} (SMRL, por sus siglas en inglés). La escala está compuesta por 11 ítems que miden la aceptación de los mitos románticos (véase Anexo I). La SMRL tiene un formato de respuesta tipo Likert con 5 alternativas: 1, completamente en desacuerdo; 2 , en desacuerdo; 3 , ni de acuerdo ni desacuerdo; 4, de acuerdo; y 5, completamente de acuerdo. Todos ítems tienen una dirección directa, por lo que las puntuaciones altas suponen mayores niveles de acuerdo. Los mitos del amor romántico pueden medirse de forma independiente a través de sus indicadores o de forma conjunta a través de la puntuación total en la escala. La estructura interna y la confiabilidad de la SMRL se analizan a lo largo del documento.

\section{Inventario de Sexismo Ambivalente} (Glick \& Fiske, 1996). Se utilizó la versión reducida y validada en castellano (Rodríguez et al., 2009a). Se trata de una escala que mide actitudes sexistas hacia las mujeres. Esta consta de 12 ítems con un formato de respuesta tipo Likert de 6 puntos ( 0 , totalmente en desacuerdo; a 5, totalmente de acuerdo). Los ítems se agrupan en dos factores de 6 ítems cada uno: sexismo hostil (e.g. Una vez que una mujer logra que un hombre se comprometa con ella, por lo general intenta controlarlo estrechamente) y sexismo benévolo (e.g. Todo hombre debe tener una mujer a quien amar). La consistencia interna de la escala en la presente investigación ha sido adecuada: sexismo hostil, $\alpha=.84$; sexismo benévolo, $\alpha=.79$.

4. Escala de Mitos sobre el Amor (Bosch et al., 2008). Se hizo uso de la versión reducida y validada en castellano (Rodríguez-Castro et al., 2013). Esta consta de 7 ítems con un formato de repuesta tipo Likert de 5 puntos (1, totalmente en desacuerdo; a 5, totalmente de acuerdo). Los ítems se agrupan en 2 dimensiones: Idealización del amor (e.g. El amor es ciego) y Vinculación amor-maltrato (e.g. Se puede amar a alguien a quien se maltrata). La consistencia interna de la escala en la presente investigación ha sido aceptable: Idealización del amor, $\alpha=.67$; Vinculación amor-maltrato, $\alpha=.75$.

\section{Procedimiento}

El diseño de la SMRL se realizó a partir de la revisión de la bibliografía y de los instrumentos previos en castellano. Para cada uno de los mitos románticos fueron creados de forma preliminar varios ítems, con el objetivo de seleccionar posteriormente los más representativos. Los ítems, redactados en estilo impersonal y en primera persona, exploraban los mitos descritos anteriormente: mito de la media naranja, mito de la pasión eterna, mito del matrimonio, mito de la pareja, mito de la omnipotencia, mito de la exclusividad, mito de la abnegación, mito de los celos y mito de la ambivalencia. Los ítems fueron diseñados pensando en que se agruparían en dos dimensiones: Mitos del Amor Idealizado y Mitos del Amor Distorsionado. Los primeros idealizan el modelo de amor romántico y los segundos legitiman los esquemas relacionales de dominación-sumisión. La evidencia de validez de contenido se evaluó a través de cuatro expertas en el campo de los Estudios de Género, que fueron seleccionadas por su experiencia y conocimiento en la materia. La escala definitiva fue administrada a 1168 estudiantes de la Comunidad de Madrid. La participación en el estudio fue voluntaria y anónima. Las personas participantes fueron informadas de los objetivos y las características de la investigación. Los datos se recogieron a través de un cuestionario autoaplicado. 


\section{Análisis}

El programa estadístico utilizado para el análisis de datos fue SPSS (IBM SPSS Statistics 22.0). El porcentaje de valores perdidos en los ítems de la SMRL osciló entre el $0 \%$ y el $0.2 \%$, por lo que fueron reemplazados por la media de las series. La estructura factorial de la SMRL se estudió de modo secuencial a través de las técnicas Análisis Factorial Exploratorio (AFE) y Análisis Factorial Confirmatorio (AFC), dividiendo aleatoriamente la muestra en dos submuestras de aproximadamente el 50\% (LloretSegura, Ferreres-Traver, Hernández-Baeza, \& Tomás-Marco, 2014). El AFE se realizó con la primera submuestra $(\mathrm{n} 1=595)$ y el $\mathrm{AFC}$ con la segunda submuestra $(\mathrm{n} 2=573)$. El índice KMO (Kaiser-Meyer-Olkin) y la prueba de esfericidad de Barlett fueron utilizados para valorar la adecuación de la matriz de correlaciones. La normalidad univariante se analizó mediante la curtosis y la asimetría, estableciendo como criterio que los valores se situasen por debajo del valor absoluto 7 y por debajo del valor absoluto 2 , respectivamente (Curran, West, \& Finch, 1996). En el AFE se extrajeron los factores con autovalores iguales o superiores a la unidad, se suprimieron los coeficientes inferiores a $.30 \mathrm{y}$ se utilizó la técnica de rotación Oblimin directo. Asimismo, de forma complementaria, se realizó un Análisis Paralelo (AP) con el programa FACTOR 10.10.03 (Lorenzo-Seva \& Ferrando, 2006), haciendo uso también de la técnica de rotación oblicua Oblimin directo. El AFC, utilizando la metodología SEM (Structural Equation Modeling), se realizó con el software estadístico AMOS (IBM AMOS 24.0). Fueron testados cuatro modelos (véase Figura 1): modelo unifactorial, modelo de dos factores independientes (ortogonales), modelo de dos factores correlacionados (oblicuos) y modelo bifactor. La calidad de ajuste de los distintos modelos se analizó en base a distintos índices de ajuste absoluto, incremental y de parsimonia. Los criterios utilizados para evaluar el ajuste de los modelos fueron los siguientes: $\chi^{2} / g l \leq 3$, GFI, AGFI y CFI $\geq .95 ; \quad$ SRMR $\leq .08 ; \quad$ RMSEA $\leq .06$ (Schreiber, Nora, Stage, Barlow, \& King, 2006), aunque valores de GFI, AGFI y CFI $\geq .90$ y de RMSEA $\leq .08$ pueden considerarse aceptables (Medrano \& Muñoz-Navarro, 2017). Para la comparación de modelos, también se utilizó el Criterio de Información de Akaike (AIC). El análisis del modelo bifactor incluyó los siguientes índices (Dominguez-Lara \& Rodríguez, 2017; Rodriguez, Reise, \& Haviland, 2016): omega jerárquico general $\left(\omega_{\mathrm{h}}\right)$ y por subescalas $\left(\omega_{\mathrm{hs}}\right)$, coeficiente $\mathrm{H}$ jerárquico $\left(\mathrm{H}_{\mathrm{h}}\right)$, ECV (Explained Common Variance) y ECV-I (ECV de los ítems), el FD (Factor Determinacy) y el ARPB (Average Relative Parameter Bias). Estos se calcularon con los módulos Índices Bifactor (Dominguez-Lara \& Rodríguez, 2017) y Bifactor Indices Calculator (Dueber, 2017). Para evaluar la confiabilidad se incluyeron el omega $(\omega)$, el coeficiente $\mathrm{H}(\mathrm{H})$ (Domínguez-Lara, 2016) y el alfa de Cronbach con intervalos de confianza (Domínguez-Lara \& Merino-Soto, 2015). A partir de los resultados del modelo bifactor, los ítems con valores altos de ECV-I fueron seleccionados para obtener una versión breve de la escala (Stucky \& Edelen, 2014). Por último, se calcularon las correlaciones entre la SMRL, el ASI y la Escala de Mitos sobre el Amor, como medida de validez convergente. Para considerar estadísticamente significativo un resultado, se adoptó la probabilidad de cometer un error tipo I de $p<.05$.

\section{Resultados}

\section{Validez de contenido}

La validez de contenido de la SMRL se evaluó mediante la técnica juicio de expertos (Boateng, Neilands, Frongillo, Melgar-Quiñonez, \& Young, 2018; Carretero-Dios \& Pérez, 2005, 2007). El juicio lo realizaron cuatro expertas en el campo de los Estudios de Género, a quienes se envió el listado preliminar de ítems y las instrucciones de la evaluación. La tarea de las expertas consistió en valorar el grado de comprensibilidad de los ítems (claridad) y el grado de correspondencia entre el contenido y el mito que se pretendía medir (pertinencia). Para ello, hicieron uso de una escala de 5 puntos en la que se expresaba el grado de claridad (1, nada claro; 5, muy claro) y el grado de pertinencia (1, nada pertinente; 5, muy pertinente). Tras la revisión de los juicios emitidos por las expertas, se descartaron los ítems que estaban redactados en estilo personal y se seleccionaron los que estaban en estilo impersonal. Entre estos, pasaron a formar 
Tabla 2. Listado final de ítems y mito que representan

\begin{tabular}{lll}
\hline $\mathrm{N}^{\mathbf{o}}$ & Formulación final de los ítems de la SMRL & Mito que evalúan \\
\hline Ítem 1 & El amor verdadero puede superar cualquier dificultad o problema & Mito de la omnipotencia \\
Ítem 2 & Todas las personas encontrarán en algún momento a su alma gemela & Mito de la media naranja \\
Ítem 3 & Cuando se encuentra a la pareja perfecta, no hay que dejarla marchar & Mito de la media naranja \\
Ítem 4 & $\begin{array}{l}\text { Una persona no será plenamente feliz hasta que no encuentre una pareja que le } \\
\text { complemente }\end{array}$ & Mito de la pareja \\
Ítem 5 & Solo es amor verdadero cuando se mantiene la pasión de los primeros meses & Mito de la pasión eterna \\
Ítem 6 & No se puede amar a más de una persona al mismo tiempo & Mito de la exclusividad \\
Ítem 7 & El amor verdadero debe terminar en matrimonio & Mito del matrimonio \\
Ítem 8 & $\begin{array}{l}\text { Una persona enamorada se preocupa más por el bienestar de su pareja que por el } \\
\text { suyo }\end{array}$ & Mito de la abnegación \\
Ítem 9 & $\begin{array}{l}\text { Amar a alguien es hacer todo por esa persona, aunque en ocasiones conlleve hacer } \\
\text { cosas que no te gustan }\end{array}$ & Mito de la abnegación \\
Ítem 10 & Los celos son una demostración de amor hacia la pareja & Mito de los celos \\
Ítem 11 & Los gritos e insultos de la pareja se pueden perdonar siempre que haya amor & Mito de la ambivalencia \\
\hline
\end{tabular}

Tabla 3. Estadísticos descriptivos y pruebas de normalidad

\begin{tabular}{lcccccc}
\hline & $\mathrm{M}$ & $\mathrm{SD}$ & $r_{\text {item-total }} \mathrm{c}^{\mathrm{c}}$ & $\alpha$-ítem & Asimetría & Curtosis \\
\hline Ítem 1 & 3.01 & 1.17 & .50 & .78 & -.12 & -.92 \\
Ítem 2 & 2.71 & 1.17 & .43 & .79 & .18 & -.81 \\
Ítem 3 & 2.95 & 1.17 & .57 & .77 & .02 & -.75 \\
Ítem 4 & 1.73 & .98 & .47 & .79 & 1.40 & 1.42 \\
Ítem 5 & 1.75 & .84 & .37 & .79 & 1.10 & 1.10 \\
Ítem 6 & 2.68 & 1.30 & .35 & .80 & .36 & -.97 \\
Ítem 7 & 2.01 & 1.07 & .54 & .78 & .90 & .21 \\
Ítem 8 & 2.61 & 1.15 & .49 & .78 & .20 & -.92 \\
Ítem 9 & 2.30 & 1.14 & .48 & .78 & .54 & -.70 \\
Ítem 10 & 1.53 & .81 & .49 & .79 & 1.50 & 1.79 \\
Ítem 11 & 1.58 & .85 & .41 & .79 & 1.57 & 2.26 \\
\hline
\end{tabular}

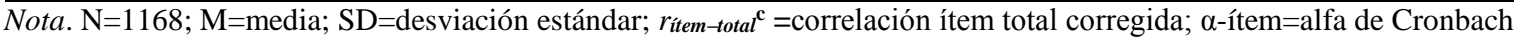
si se suprime el elemento.

parte de la escala los que, teniendo una puntuación media en claridad y pertinencia igual o superior a 4, representaban mejor los mitos románticos. La SMRL finalmente quedó compuesta por 11 ítems. La puntuación media de los once ítems seleccionados fue 4.50 en claridad $(\mathrm{SD}=.42)$ y 4.64 en pertinencia $(\mathrm{SD}=.34)$. A partir de las observaciones de las expertas, a su vez, fue modificada la redacción de algunos ítems. La Tabla 2 muestra la formulación final de los ítems y el mito que representa cada uno.

\section{Análisis descriptivos y pruebas de normalidad}

La Tabla 3 muestra los estadísticos descriptivos y las pruebas de normalidad de los 11 ítems que componen la SMRL. Las puntuaciones en la desviación estándar resultaron próximas a la unidad y las medias oscilaron entre 1.53 y 3.01 . Los coeficientes de la correlación ítem total corregida fueron superiores a .30 y el alfa de Cronbach de la escala $(\alpha=.80$, IC 95\% = .78 - .82) no mejoraba con la eliminación de elementos. Los valores de asimetría oscilaron entre -.12 y 1.57 y los valores de curtosis entre -.97 y 2.26 , por lo que los datos estuvieron dentro de los rangos de normalidad univariante (Curran et al, 1996). Por lo tanto, partiendo de los resultados obtenidos, los distintos análisis factoriales de la SMRL se realizaron con el método de extracción Máxima Verosimilitud.

\section{Análisis Factorial Exploratorio}

El AFE se realizó sobre la primera submuestra (n1=595). Para calcular el grado de adecuación de muestreo, antes de analizar la dimensionalidad de la escala, se calcularon el Índice de Kaiser-MeyerOlkin y la prueba de esfericidad de Bartlett. Los valores obtenidos en ambas pruebas $(\mathrm{KMO}=.871$; $\chi^{2}$ Esfericidad $\left.=1246.27, g l=55, p<.001\right)$ fueron adecuados (Montoya, 2007), por lo que se procedió a analizar la estructura factorial de la SMRL. El AFE arrojó una estructura de dos factores cuyos autovalores iniciales eran iguales o superiores a la unidad: 3.68 en el primer factor y 1.16 en el segundo. Las dos dimensiones, en su conjunto, explicaban el $44.01 \%$ de la varianza. La 
Tabla 4. Pesos factoriales del Análisis Paralelo

\begin{tabular}{lccc}
\hline & $\begin{array}{c}\text { Matriz factorial rotada del modelo de } \\
\text { dos factores }\end{array}$ & $\begin{array}{c}\text { Matriz factorial del modelo } \\
\text { unidimensional }\end{array}$ \\
\cline { 2 - 4 } & Factor 1 & Factor 2 & Factor único \\
\hline Ítem 1 & .539 & .567 \\
Ítem 2 & .657 & .452 \\
Ítem 3 & .574 & .612 \\
Ítem 4 & .358 & & .543 \\
Ítem 5 & .355 & & .437 \\
Ítem 6 & .442 & & .418 \\
Ítem 7 & .347 & .431 & .577 \\
Ítem 8 & & .504 & .535 \\
Ítem 9 & & .544 & .512 \\
Ítem 10 & & .663 & .564 \\
Ítem 11 & & .456 \\
\hline
\end{tabular}

Nota. $\mathrm{N}=595$. La tabla omite las cargas inferiores a .30; F1=Mitos del Amor Idealizado; F2=Mitos del Amor Distorsionado; Factor único=Mitos Románticos.

Tabla 5. Índices de bondad de ajuste de los distintos modelos analizados

\begin{tabular}{ccccccccccc}
\hline & $\chi^{2}$ & $\mathrm{gl}$ & $p$ & $\chi^{2} / \mathrm{gl}$ & GFI & AGFI & CFI & RMSEA [IC 90\%] & SRMR & AIC \\
\hline M1 & 145.32 & 44 & .000 & 3.30 & .96 & .93 & .92 & $.06[.05-.08]$ & .04 & 189.32 \\
M2 & 362.16 & 44 & .000 & 8.23 & .91 & .87 & .76 & $.11[.10-.12]$ & .18 & 406.16 \\
M3 & 110.87 & 43 & .000 & 2.58 & .97 & .95 & .95 & $.05[.04-.07]$ & .04 & 156.87 \\
M4 & 57.07 & 33 & .006 & 1.73 & .98 & .97 & .98 & $.04[.02-.05]$ & .03 & 123.07 \\
\hline
\end{tabular}

Nota. $\mathrm{N}=573 ; \mathrm{M} 1=$ modelo de un factor; $\mathrm{M} 2=$ modelo de dos factores independientes; $\mathrm{M} 3=$ =modelo de dos factores correlacionados; M4=modelo bifactor.

solución factorial rotada mostró que el Factor 1 estaba compuesto por siete ítems y el Factor 2, por cuatro ítems. No obstante, si bien la regla de Kaiser apuntaba a una estructura interna de dos componentes, se realizó un AP como medida complementaria para comprobar si el número de factores a retener era el adecuado (Horn. 1965).

En el AP, partiendo de la solución rotada obtenida en el AFE, se probó en primer lugar una estructura de dos factores. La distribución de los ítems en la matriz factorial rotada fue la misma que la del AFE, con cargas factoriales que oscilaban entre .347 y .657 en el primer factor y entre .431 y .663 en el segundo (véase Tabla 4). Todos estadísticos de ajuste fueron adecuados: $\mathrm{GFI}=.99$; AGFI=.99; CFI=.99; RMSEA=.042; RMSR=.031. Sin embargo, el AP aconsejaba probar una estructura unidimensional, por lo que se repitió el análisis para un modelo de un solo factor. En la misma línea que el modelo anterior, la calidad de ajuste también fue adecuada (GFI=.98; AGFI=.98; $\mathrm{CFI}=.95 ; \mathrm{RMSEA}=.065$; $\mathrm{RMSR}=.052$ ) y las cargas factoriales oscilaron entre .418 y .612 .

\section{Análisis Factorial Confirmatorio}

El siguiente paso fue contrastar la dimensionalidad mediante un AFC sobre la segunda submuestra (n2=573). Los análisis anteriores planteaban tres posibles estructuras: un modelo de un factor, un modelo de dos factores independientes y un modelo de dos factores correlacionados. La Tabla 5 recoge los índices de ajuste absoluto, incremental y de parsimonia de los modelos analizados. Los resultados reportaron un ajuste inadecuado para el modelo de dos factores independientes, pero aceptable para los modelos de un factor y de dos factores correlacionados, siendo mejor en el último de ellos $\left(\Delta \chi^{2}=-34.45 ; \Delta g l=-1 ; \Delta \chi^{2} / g l=-.72 ; \Delta \mathrm{AIC}=-32.45\right)$. Los parámetros estimados de ambos modelos fueron significativos $(p<.001)$ y las cargas estandarizadas, superiores a .30. Sin embargo, aunque los índices de ajuste parecían indicar que tanto la estructura unidimensional como la multidimensional eran consistentes, la correlación interfactorial del modelo de dos factores oblicuos fue elevada ( $\Phi=.83$ ), lo que podía ser un indicador de información redundante y superposición factorial. En este sentido, haciendo uso del modelamiento bifactor (Domínguez-Lara \& Rodríguez, 2017; Rodriguez et al., 2016), se puso a prueba un cuarto modelo para evaluar de forma simultánea la presencia de un factor general y de dos factores específicos modelados ortogonalmente. La Figura 1 ilustra los cuatro modelos analizados. Los resultados mostraron que la bondad de ajuste del modelo bifactor fue óptima (Schreiber et al., 2006), mejor que la del 

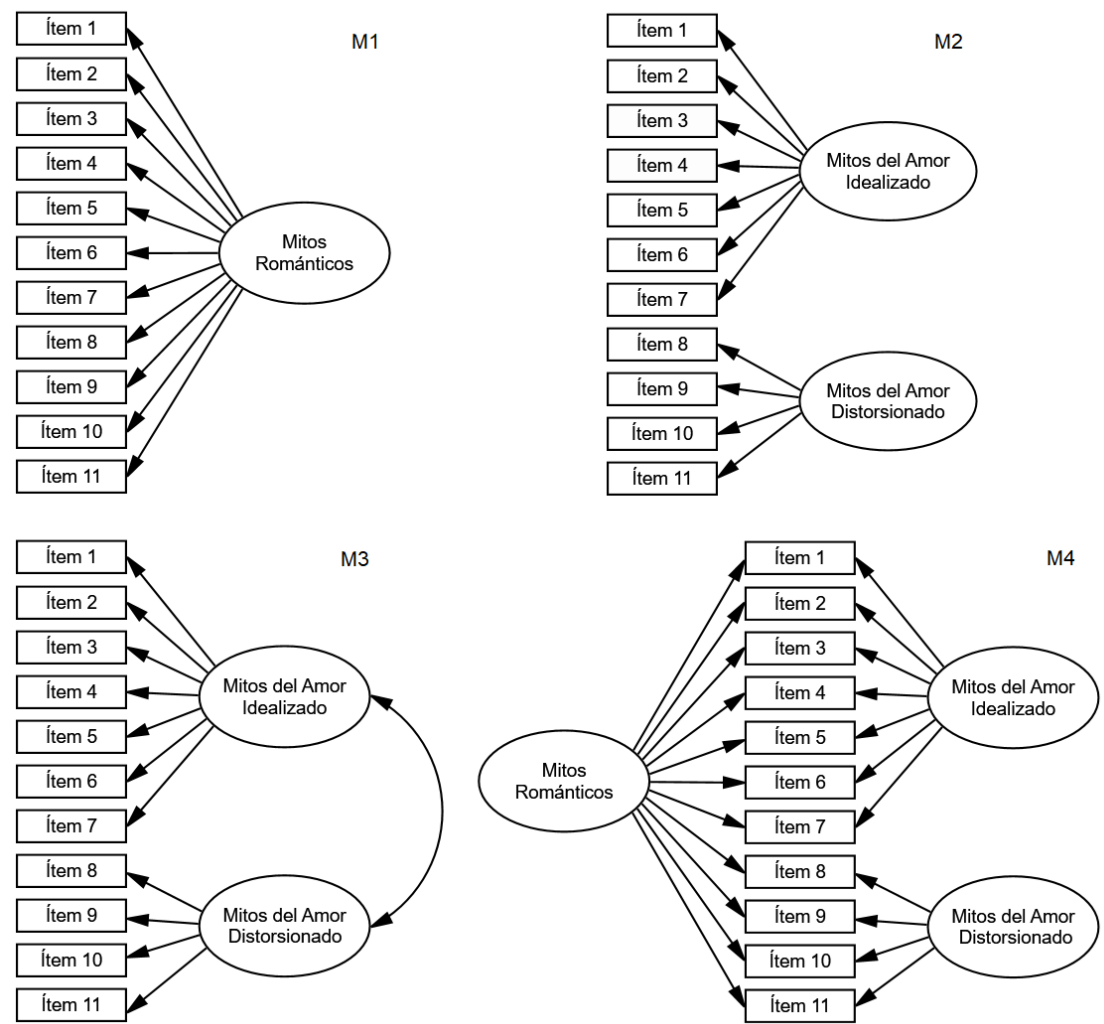

Figura 1. Modelos analizados mediante AFC

Nota. M1=modelo unifactorial; M2=modelo de dos factores independientes; M3=modelos de dos factores correlacionados; M4=modelo bifactor. Los errores han sido eliminados para facilitar la comprensión de los modelos.

modelo de un factor $\left(\Delta \chi^{2}=-88.25 ; \Delta g l=-11\right.$; $\left.\Delta \chi^{2} / g l=-1.57 ; \Delta \mathrm{AIC}=-66.25\right)$ y que la del modelo de dos factores correlacionados $\left(\Delta \chi^{2}=-53.08\right.$; $\left.\Delta g l=-10 ; \Delta \chi^{2} / g l=-.85 ; \Delta \mathrm{AIC}=-33.8\right)$.

Los análisis bifactor no deben basarse únicamente en los índices de ajuste porque, aunque los modelos tengan un ajuste óptimo, se desconoce el grado de influencia del factor general sobre los ítems, en comparación con los factores específicos (Domínguez-Lara \& Rodríguez, 2017; Flores-Kanter, DomínguezLara, Trógolo, \& Medrano, 2018; Rodriguez et al., 2016); de manera que se analizaron varios estadísticos adicionales para evaluar la dimensionalidad de la SMRL (véase Tabla 6). Los resultados del modelo bifactor reportaron un coeficiente omega aceptable tanto en el factor general $(\omega>.80)$ como en los factores específicos $\left(\omega_{\mathrm{s}}>.70\right)$. El FD (.90), el ECV (.74) y el coeficiente $H$ jerárquico $\left(\mathrm{H}_{\mathrm{h}}>.80\right)$ reportaron evidencias de la fortaleza del factor general, en contraste con los dos factores específicos $\left(\mathrm{ECV}_{\mathrm{s} 1}=.10 ; \mathrm{ECV}_{\mathrm{s} 2}=.16 ; \mathrm{FD}_{\mathrm{s} 1}=.69 ; \mathrm{FD}_{\mathrm{s} 2}=.75 ; \mathrm{H}_{\mathrm{hs} 1}\right.$ y $\left.\mathrm{H}_{\mathrm{hs} 2}<.60\right)$. El factor general explicó la mayor parte de la varianza del puntaje total $\left(\omega_{\mathrm{h}}>70\right)$, mientras que, cuando se controló la variabilidad debida al factor general, los dos factores específicos explicaron un bajo porcentaje de la varianza confiable $\left(\omega_{\mathrm{hs}}<.30\right)$, lo que indica que la interpretación de las dos dimensiones como constructos únicos sería limitada porque los ítems están influidos esencialmente por el factor general y solo trivialmente por los factores específicos. Los ítems del modelo bifactor, en promedio, recibían una mayor influencia del factor general que de los factores específicos $\left(\mathrm{ECV}-\mathrm{I}_{\text {promedio }}=\right.$ .821 ), con el $72.7 \%$ de los ítems con un ECV-I superior a .80 , de manera que hay muy poca varianza verdadera a nivel de ítems más allá de la que es atribuible al factor general. Por último, el sesgo relativo promedio fue inferior al $10 \%$ (ARPB=.051), lo que indica que las diferencias entre las cargas factoriales del modelo unidimensional y las del factor general del bifactor son pequeñas. En conclusión, los análisis estructurales y los índices adicionales del modelo bifactor brindan evidencias a favor de una concepción unidimensional y no multidimensional de la SMRL. 
Tabla 6. Cargas factoriales estandarizadas y confiabilidad

\begin{tabular}{|c|c|c|c|c|c|c|c|c|c|c|c|c|}
\hline & \multicolumn{2}{|c|}{ Modelo unifactorial } & \multicolumn{3}{|c|}{$\begin{array}{l}\text { Modelo de dos } \\
\text { factores } \\
\text { independientes }\end{array}$} & \multicolumn{3}{|c|}{$\begin{array}{l}\text { Modelo de dos } \\
\text { factores oblicuos }\end{array}$} & \multicolumn{4}{|c|}{ Modelo bifactor } \\
\hline & $F$ & $\mathrm{R}^{2}$ & F1 & $\mathrm{F} 2$ & $\mathrm{R}^{2}$ & F1 & F2 & $\mathrm{R}^{2}$ & FE1 & FE2 & FG & $\mathrm{R}^{2}$ \\
\hline Ítem 1 & .553 & .306 & .584 & & .341 & .572 & & .328 & .600 & & .530 & .641 \\
\hline Ítem 2 & .508 & .258 & .553 & & .306 & .532 & & .283 & .187 & & .499 & .284 \\
\hline Ítem 3 & .661 & .437 & .692 & & .479 & .683 & & .467 & .153 & & .661 & .460 \\
\hline Ítem 4 & .522 & .273 & .522 & & .273 & .531 & & .282 & -.059 & & .553 & .310 \\
\hline Ítem 5 & .372 & .138 & .389 & & .151 & .383 & & .147 & .002 & & .380 & .144 \\
\hline Ítem 6 & .365 & .133 & .386 & & .149 & .377 & & .142 & .040 & & .373 & .141 \\
\hline Ítem 7 & .635 & .403 & .604 & & .365 & .639 & & .408 & -.065 & & .659 & .439 \\
\hline Ítem 8 & .588 & .346 & & .554 & .307 & & .618 & .382 & & .135 & .568 & .341 \\
\hline Ítem 9 & .576 & .332 & & .534 & .285 & & .600 & .360 & & .150 & .557 & .333 \\
\hline Ítem 10 & .557 & .310 & & .659 & .434 & & .612 & .375 & & .376 & .502 & .393 \\
\hline Ítem 11 & .507 & .257 & & .664 & .441 & & .577 & .332 & & .686 & .426 & .652 \\
\hline$\omega$ & .814 & & .738 & .697 & & .736 & .694 & & .755 & .726 & .837 & \\
\hline$\omega_{\mathrm{h}}$ & & & & & & & & & .039 & .218 & .776 & \\
\hline $\mathrm{H}$ & .826 & & .761 & .706 & & .761 & .695 & & & & & \\
\hline $\mathrm{H}_{\mathrm{h}}$ & & & & & & & & & .387 & .523 & .819 & \\
\hline$\lambda_{\text {promedio }}$ & .531 & & .533 & .603 & & .531 & .602 & & .123 & .337 & .519 & \\
\hline
\end{tabular}

Nota. $\mathrm{N}=573 ; \mathrm{F}=$ factor de Mitos Románticos; $\mathrm{R}^{2}=$ coeficientes de determinación; F1=Mitos del Amor Idealizado; F2= Mitos del Amor Distorsionado; FE1=factor específico de Mitos del Amor Idealizado; FE2=factor específico de Mitos del Amor Distorsionado; $\mathrm{FG}=$ factor general de Mitos Románticos; $\omega=$ coeficiente omega; $\omega_{\mathrm{h}}=$ coeficiente omega jerárquico; $\mathrm{H}=$ coeficiente $\mathrm{H}$; $\mathrm{H}_{\mathrm{h}}=$ coeficiente $\mathrm{H}$ jerárquico; $\lambda_{\text {promedio }}=$ carga factorial promedio.

Tabla 7. Medidas de validez convergente

\begin{tabular}{|c|c|c|c|}
\hline & $\begin{array}{c}\text { Mitos sobre el amor } \\
\text { (Rodríguez-Castro et al., 2013) }\end{array}$ & $\begin{array}{c}\text { Sexismo hostil } \\
\text { (Rodríguez et al., 2009a) }\end{array}$ & $\begin{array}{c}\text { Sexismo benévolo } \\
\text { (Rodríguez et al., 2009a) }\end{array}$ \\
\hline Ítem 1 & $.49 * * *$ & $.21 * * *$ & $.31^{* * *}$ \\
\hline Ítem 2 & $.54 * * *$ & $.23 * * *$ & $.34 * * *$ \\
\hline Ítem 3 & $.45^{* * *}$ & $.27 * * *$ & $.36 * * *$ \\
\hline Ítem 4 & $.33 * * *$ & $.27 * * *$ & $.39 * * *$ \\
\hline Ítem 5 & $.29 * * *$ & $.14 * * *$ & $.21 * * *$ \\
\hline Ítem 6 & $.24 * * *$ & $.15^{* * *}$ & $.24 * * *$ \\
\hline Ítem 7 & $.37 * * *$ & $.32 * * *$ & $.43 * * *$ \\
\hline Ítem 8 & $.40 * * *$ & $.27 * * *$ & $.34 * * *$ \\
\hline Ítem 9 & $.33 * * *$ & $.31 * * *$ & $.34 * * *$ \\
\hline Ítem 10 & $.48 * * *$ & $.33 * * *$ & $.41 * * *$ \\
\hline Ítem 11 & $.28 * * *$ & $.28 * * *$ & $.31 * * *$ \\
\hline Puntuación SMRL & $.66^{* * *}$ & $.43 * * *$ & $.57 * * *$ \\
\hline Puntuación B-SMRL & $.56^{* * *}$ & $.40 * * *$ & $.53 * * *$ \\
\hline
\end{tabular}

\section{Versión breve de la SMRL}

Partiendo de los resultados obtenidos en el modelo bifactor, fueron seleccionados los ítems más representativos del factor general y menos representativos de los factores específicos, a fin de crear una versión breve y unifactorial (B-SMRL). Los elementos con valores altos de ECV-I son buenos candidatos para estos objetivos (Stucky \& Edelen, 2014), por lo que pasaron a formar parte de la B-SMRL los ítems del 3 al 9, cuyos valores de ECV-I fueron superiores a .90. El AFC y las pruebas de confiabilidad se realizaron con el número total de participantes $(\mathrm{n}=1168)$. El modelo breve de siete ítems presentó índices de ajuste óptimos $\quad\left(\chi^{2}{ }_{(14)}=48.87 ; \quad p<.001 ; \quad\right.$ GFI $=.99$; AGFI=.98; CFI=.97; RMSEA $=.05$ [IC 90\%=.03 -
.06]; SRMR=.03). La carga factorial promedio fue .532 , con pesos estandarizados entre .400 y .633 , $\mathrm{y}$ todos los estimadores resultaron estadísticamente significativos $\quad(p<.001)$. En cuanto a la confiabilidad de los puntajes y del constructo, tanto el alfa de Cronbach $(\alpha=.73$, IC $95 \%=.70-.76)$ como los coeficientes $\mathrm{H}$ y omega $(\mathrm{H}=.75 ; \omega=.74)$ fueron aceptables.

\section{Validez convergente}

Por último, como medida de validez convergente, se analizaron las correlaciones lineales de Pearson entre la SMRL, la Escala de Mitos sobre el Amor (Rodríguez-Castro et al., 2013) y el Inventario de Sexismo Ambivalente (Rodríguez et al., 2009a). Los resultados 
reportaron que las correlaciones con los mitos sobre el amor $(r=.66)$ y con el sexismo ambivalente $(r=.57)$ fueron estadísticamente significativas $(p<.001)$ y se produjeron en la dirección esperada, lo que evidenció la validez convergente de la escala. Asimismo, tal y como muestra la Tabla 7, las puntuaciones altas en los mitos románticos estarían relacionadas con las puntuaciones altas en las actitudes sexistas; todos los mitos del amor romántico que componen la SMRL estuvieron significativamente relacionados con el sexismo hostil, el sexismo benévolo y los mitos sobre el amor. La B-SMRL, en la misma línea, estuvo relacionada con ambos constructos.

\section{Discusión}

En las últimas décadas, las dinámicas relacionales y amorosas se han convertido en un creciente foco de interés para las ciencias sociales (Cuenca-Montesino, Graña, \& O’Leary, 2015; García \& Cedillo, 2011). Las relaciones afectivas tienen una gran importancia a lo largo de las diferentes etapas del ciclo vital (Penagos, Rodríguez, Carrillo, \& Castro, 2006; RodríguezCastro et al., 2013) y el amor, como constructo cultural, está íntimamente relacionado con las estructuras sociales y los periodos sociohistóricos (Bonilla \& Rivas, 2018; Ferrer et al., 2008; García et al., 2019; García \& Montenegro, 2014; Lagarde, 2001; Rocha et al., 2017; Schäfer, 2008). En este sentido, si las prácticas de la vida íntima están relacionadas con las prácticas de la vida social (García \& Montenegro, 2014: Schäfer, 2008), el estudio del amor romántico tiene que atender a las condiciones sociales, culturales, históricas, políticas y económicas que lo enmarcan. El objetivo de esta investigación ha sido diseñar y validar en el contexto español un nuevo instrumento que permita profundizar en el análisis de los distintos mitos del amor romántico y sus implicaciones en las relaciones íntimas.

La validez de contenido de la escala fue evaluada a partir del juicio de varias expertas en la materia (Boateng et al., 2018; Carretero-Dios \& Pérez, 2005; 2007). Las puntuaciones medias de los ítems seleccionados fueron altas tanto en claridad como en pertinencia, por lo que estos representan correctamente los mitos del amor romántico. Los datos estuvieron dentro de la curva normal univariante (Curran et al, 1996) y los coeficientes de la correlación ítem total corregida fueron superiores a .30 (Boateng et al., 2018; Carretero-Dios \& Pérez, 2005). El alfa de Cronbach de la SMRL no mejoraba con la eliminación de elementos $\mathrm{y}$, junto a los intervalos de confianza (Dominguez-Lara \& Merino-Soto, 2015), presentó valores aceptables $(\alpha>$.70; Campo-Arias \& Oviedo, 2008).

La dimensionalidad de la SMRL se analizó a través de una técnica secuencial, aplicando un AFE sobre la mitad de la muestra y un AFC sobre la otra mitad (Lloret-Segura et al., 2014). El AFE, utilizando la regla de Kaiser y rotación oblicua, reportó la existencia de dos factores con valores propios mayores que la unidad. No obstante, aunque los resultados de un AP complementario mostraron que el ajuste del modelo de dos factores era bueno, el análisis sugirió retener únicamente un factor. Un nuevo AP, tal y como se esperaba, reportó que la calidad de ajuste del modelo unidimensional también era adecuada. Estos resultados abrieron varias posibilidades estructurales para la SMRL, que fueron analizadas mediante un análisis factorial confirmatorio.

Los modelos de un factor y de dos factores correlacionados obtuvieron índices aceptables de ajuste absoluto, incremental y de parsimonia (Medrano \& Muñoz-Navarro, 2017). Los índices del modelo oblicuo fueron mejores, sin embargo, dada la elevada correlación interfactorial $(\Phi>$ .80), se puso a prueba un modelo bifactor para comprobar en qué medida esta relación se podía explicar por la presencia de un factor general (Dominguez-Lara \& De la Cruz-Contreras, 2017; Domínguez-Lara \& Rodríguez, 2017). El modelo bifactor de la SMRL planteaba la presencia simultanea de un factor general (Mitos Románticos) y dos factores específicos (Mitos del Amor Idealizado y Mitos del Amor Distorsionado) modelados ortogonalmente. Los resultados del AFC reflejaron que el modelo presenta mejor ajuste que los otros modelos analizados, con todos los índices por encima de los puntos de corte (Schreiber et al., 2006). No obstante, si bien el factor general es lo suficientemente fuerte como ser considerado, los estadísticos adicionales del modelo bifactor reportaron que los dos factores específicos son en gran medida redundantes con el factor general y 
no proporcionan información sustancial más allá de este.

La varianza común explicada general (ECV>.70) y de los ítems (ECV-I $\left.\mathrm{I}_{\text {promedio }}>.80\right)$, el coeficiente $\mathrm{H}$ jerárquico $\left(\mathrm{H}_{\mathrm{h}}>.80\right)$ y el omega jerárquico $\left(\omega_{\mathrm{h}}>\right.$.70) evidenciaron que el factor general es más robusto que los factores específicos y que la varianza de los indicadores es atribuible principalmente al factor general (FloresKanter et al., 2018). En este sentido, cuando la influencia del factor general fue controlada, los factores específicos explicaron un porcentaje no significativo de la varianza confiable $\left(\omega_{\mathrm{hs}}<.30\right)$ (Smits, Timmerman, Barelds, \& Meijer, 2015) y obtuvieron coeficientes $\mathrm{H}$ bajos $\left(\mathrm{H}_{\mathrm{hs}}<.60\right)$ (Domínguez-Lara \& Rodríguez, 2017; Rodriguez et al., 2016). Las cargas del modelo unidimensional apenas difirieron de las del factor general del bifactor (ARPB>.10) (Rodriguez et al., 2016). En conclusión, tomando en consideración los estadísticos de bondad de ajuste del AFC y los índices adicionales del modelo bifactor, los resultados apoyan que la SMRL es principalmente unidimensional.

La versión breve de la escala (B-SMRL) también cuenta con respaldo empírico. Los ítems fueron seleccionados según sus valores de ECV-I, garantizando la unidimensionalidad de la medida (Stucky \& Edelen, 2014). El modelo presentó índices de ajuste adecuados (Medrano \& MuñozNavarro, 2017; Schreiber et al., 2006) y todos los estimadores fueron significativos. El alfa de Cronbach y sus intervalos de confianza reportaron una fiabilidad aceptable $(\alpha>$.70; Campo-Arias \& Oviedo, 2008; Dominguez-Lara \& Merino-Soto, 2015). Los coeficientes omega y $\mathrm{H}$, del mismo modo, brindaron soporte en apoyo de la confiabilidad del constructo (Domínguez-Lara, 2016).

Las correlaciones entre la SMRL y la Escala de Mitos hacia el Amor (Rodríguez-Castro et al., 2013) fueron directas y significativas, demostrando que la escala mide aquello para lo que fue diseñada. Por otra parte, en línea con los resultados obtenidos en distintas investigaciones nacionales e internacionales, los mitos del amor romántico correlacionaron con el sexismo ambivalente (Bonilla \& Rivas, 2018; Carrascosa et al., 2019; Nava-Reyes et al., 2018; RodríguezCastro et al., 2013; Rodríguez et al., 2015). Las relaciones fueron más fuertes con el sexismo benévolo que con el sexismo hostil, lo que podría deberse a que el primero idealiza como objetos amorosos a las mujeres que aceptan las relaciones de poder y los roles de género, mientras que el segundo rechaza mediante la coerción a las mujeres que cuestionan y desafían estos supuestos (Expósito et al., 1998; Glick et al., 2016; Glick \& Fiske, 1996; Lee et al., 2010; Overall \& Hammond, 2018; Rodríguez et al., 2009b). La actuación conjunta de ambas ideologías (amor romántico y sexismo) fomentaría las prácticas relacionales sobre las que se asientan las relaciones de género y reproduciría la subordinación de las mujeres en las relaciones íntimas heterosexuales.

La SMRL ha sido diseñada para medir un conjunto de mitos o creencias sobre el amor que representan e idealizan el modelo de amor romántico y que, en nombre del amor, justifican los esquemas de dominación-sumisión y el uso de la violencia en las relaciones íntimas. Los mitos que pueden medirse a través de la SMRL son los siguientes: mito de la media naranja, mito de la pareja, mito de la pasión eterna, mito de la omnipotencia, mito de la exclusividad, mito del matrimonio, mito de la abnegación, mito de los celos y mito de la ambivalencia. Todas estas creencias están relacionadas entre sí y, como se evidenció en los análisis factoriales, presentan un fuerte nexo (la ideología del amor romántico) que las agrupa en una dimensión común denominada Mitos Románticos. En consecuencia, la medición de los mitos del amor romántico puede hacerse a través de la puntuación total en la escala o, si lo que se quiere es analizar los mitos individualmente, pueden calcularse las puntuaciones de los indicadores.

El trabajo presenta algunas limitaciones que cabe tener en cuenta. Por un lado, la validación de la SMRL, al igual que otros estudios psicométricos realizados en España y Latinoamérica (Calleja \& Mason, 2020; SánchezPrada, Delgado-Álvarez, Bosch-Fiol, \& FerrerPérez, 2019), se realizó sobre una muestra de estudiantes universitarios y no sobre una muestra de la población general. No obstante, el tamaño muestral es grande y permite realizar este tipo de análisis multivariantes (MacCallum, Widaman, Zhang, \& Hong, 1999; Ruiz, Pardo, \& San 
Martín, 2010). Por otro lado, en la investigación participó un mayor porcentaje de mujeres que de hombres, lo que puede deberse a la división sexual del trabajo y la feminización de las titulaciones en Ciencias de la Educación. En el futuro sería recomendable llevar a cabo un análisis de invarianza entre hombres y mujeres. Por último, queda pendiente analizar la estructura interna y las propiedades psicométricas de la escala en otros países y regiones, comprobando si la dimensionalidad se mantiene estable en contextos culturales diversos.

La tenencia de una herramienta como la SMRL, que ha demostrado ser válida y fiable en el contexto español, permitirá analizar la construcción de la ideología del amor romántico y, más concretamente, las implicaciones de los mitos románticos en las relaciones íntimas. Es posible evaluar la vinculación con otros constructos que pudieran estar relacionados con los mitos o mediados por estos. No obstante, su uso no tiene por qué restringirse al ámbito de la investigación, pudiendo ser especialmente útil para los y las profesionales de la educación, particularmente para el profesorado de las etapas obligatorias, ya que la detección e identificación de este tipo de creencias puede orientar las intervenciones educativas. La literatura académica expone que la coeducación y los programas de sensibilización son fundamentales para transformar las dinámicas relacionales que son producto de la ideología romántica y del orden social patriarcal (Bonilla-Algovia \& Rivas-Rivero, 2018, 2019; Carbonell \& Mestre, 2018; Caro \& Monreal, 2017; Carrascosa et al., 2019; Ferrer \& Bosch, 2013). Por lo tanto, la información obtenida a través de la SMRL puede facilitar la realización de diagnósticos educativos y la puesta en marcha de intervenciones que fomenten una reformulación del imaginario amoroso, alejándolo de los mitos románticos y de las actitudes sexistas, y promuevan la igualdad y la no violencia.

\section{Referencias}

Barrón, A., Martínez-Íñigo, D., De Paúl, P., \& Yela, C. (1999). Romantic beliefs and myths in Spain. The Spanish Journal of Psychology, 2, 64-73.

doi:http://dx.doi.org/10.1017/S1138741600005461
Blanco, M. A. (2014). Implicaciones del uso de las redes sociales en el aumento de la violencia de género en adolescentes. Comunicación y Medios, 30, 124-141.

Boateng, G. O., Neilands, T. B., Frongillo, E. A., Melgar-Quiñonez, H. R., \& Young, S. L. (2018). Best practices for developing and validating scales for health, social, and behavioral research: A primer. Frontiers in Public Health, 6(149), 1-18. doi:https://doi.org/10.3389/fpubh.2018.00149

Bonilla, E., \& Rivas, E. (2018). Propiedades psicométricas de la versión reducida de la Escala de Mitos sobre el Amor en una muestra de estudiantes colombianos. Revista Suma Psicológica, 25(2), 162-170. doi:http://dx.doi.org/10.14349/sumapsi.2018.v25.n2 .8

Bonilla-Algovia, E., \& Rivas-Rivero, E. (2018). Mitos del amor romántico en una muestra de futuros y futuras docentes. Revista de Estudios e Investigación en Psicología y Educación, 5(2), 113-117. doi:https://doi.org/10.17979/reipe.2018.5.2.3624

Bonilla-Algovia, E., \& Rivas-Rivero, E. (2019). Creencias distorsionadas sobre la violencia contra las mujeres en docentes en formación de Colombia. Revista Colombiana de Educación, 1(77), 1-20. doi:https://doi.org/10.17227/rce.num77-9571

Bonilla-Algovia, E., \& Rivas-Rivero, E. (en prensa). Creencias sobre el amor romántico y las relaciones íntimas: Implicaciones en jóvenes de la Comunidad de Madrid. Informes Psicológicos.

http://hdl.handle.net/10017/42932

Bosch, E., Ferrer, V. A., García, M. E., Ramis, M. C., Mas, M. C., Navarro, C., \& Torrens, G. (2008). Del mito del amor romántico a la violencia contra las mujeres en la pareja. Madrid: Instituto de la Mujer.

Bosch, E., Herrezuelo, R., \& Ferrer, V.A. (2019). El amor romántico, como renuncia y sacrificio: ¿Qué opinan los y las jóvenes? Femeris, 4(3), 184-202. https://doi.org/10.20318/femeris.2019.4935

Bourdieu, P. (2010). La dominación masculina y otros ensayos. Buenos Aires: Editorial La Página. 
Bourdieu, P. (1990). The logic of practice. California: Stanford University Press.

Calleja, N., \& Mason, T.A. (2020). Escala de Bienestar Subjetivo (EBS-20 y EBS-8): Construcción y validación. Revista Iberoamericana de Diagnóstico y Evaluación - e Avaliação Psicológica, 55(2), 185-201. doi:https://doi.org/10.21865/RIDEP55.2.14

Campo-Arias, A., \& Oviedo, H.C. (2008). Propiedades psicométricas de una escala: La consistencia interna. Revista de Salud Pública, 10(5), 831-839.

Carbonell, Á., \& Mestre, M. V. (2018). Sexismo y mitos del amor romántico en estudiantes prosociales y antisociales. Revista Prisma Social, (23), 1-17.

Caro, C., \& Monreal, M. C. (2017). Creencias del amor romántico y violencia de género. International Journal of Developmental and Educational Psychology, 2(1), 47-56. doi:https://doi.org/10.17060/ijodaep.2017.n1. v2.917

Carrascosa, L., Cava, M. J., Buelga, S., \& De Jesus, S.N. (2019). Reduction of sexist attitudes, romantic myths, and aggressive behaviors in adolescents: Efficacy of the DARSI program. Psicothema. 31(2), 121-127. doi:https://doi.org/10.7334/psicothema2018.245

Carretero-Dios, H., \& Pérez, C. (2005). Normas para el desarrollo y revisión de estudios instrumentales. International Journal of Clinical and Health Psychology, 5(3), 521551.

Carretero-Dios, H., \& Pérez, C. (2007). Normas para el desarrollo y revisión de estudios instrumentales: Consideraciones sobre la selección de tests en la investigación psicológica. International Journal of Clinical and Health Psychology, 7(3), 863-882.

Connolly, P., \& Healy, J. (2004). Symbolic violence, locality and social class: The educational and career aspirations of 10-11year-old boys in Belfast. Pedagogy, Culture \& Society, 12(1), 15-33. doi:https://doi.org/10.1080/14681360400200187

Cross, E. J., \& Overall, N. C. (2018). Women's attraction to benevolent sexism: Needing relationship security predicts greater attraction to men who endorse benevolent sexism.
European Journal of Social Psychology, 48(3), 336-347.

doi:https://doi.org/10.1002/ejsp.2334

Cubells, J., \& Calsamiglia, A. (2015). El repertorio del amor romántico y las condiciones de posibilidad para la violencia machista. Universitas Psychologica, 14(5), 1681-1694.

doi:http://dx.doi.org/10.11144/Javeriana.upsy 14-5.rarc

Cuenca-Montesino, M. L., Graña, J. L., \& O'Leary, K. D. (2015). Intensity of love in a community sample of Spanish couples in the region of Madrid. The Spanish Journal of Psychology, 18(e79), 1-9. doi:https://doi.org/10.1017/sjp.2015.79

Curran, P. J., West, S. G., \& Finch, J. F. (1996). The robustness of test statistics to nonnormality and specification error in confirmatory factor analysis. Psychological Methods, 1(1), 16-29.

doi:http://dx.doi.org/10.1037/1082-989X.1.1.16

Domínguez-Lara, S. (2016). Evaluación de la confiabilidad del constructo mediante el coeficiente $\mathrm{H}$ : breve revisión conceptual y aplicaciones. Psychologia: Avances de la Disciplina, 10(2), 87-94. doi:https://doi.org/10.21500/19002386.2134

Dominguez-Lara, S., \& De la Cruz-Contreras, F. (2017). Análisis estructural y desarrollo de una versión breve de la versión en español del Inventario de Ansiedad ante Exámenes (TAIE) en universitarios de Lima. Interacciones, 3(1), 7-17. doi:https://doi.org/10.24016/2017.v3n1.50

Domínguez-Lara, S. A., \& Merino-Soto, C. (2015). ¿Por qué es importante reportar los intervalos de confianza del coeficiente alfa de Cronbach? Revista Latinoamericana de Ciencias Sociales, Niñez y Juventud, 13(2), 1326-1328.

Domínguez-Lara, S., \& Rodríguez, A. (2017). Índices estadísticos de modelos bifactor. Interacciones, 3(2), 59-65. doi:https://doi.org/10.24016/2017.v3n2.51

Dueber, D. M. (2017). Bifactor indices calculator: A Microsoft Excel-based tool to calculate various indices relevant to bifactor CFA models. doi:https://dx.doi.org/10.13023/edp.tool.01 
Esteban, M. L., \& Távora, A. (2008). El amor romántico y la subordinación social de las mujeres: Revisiones y propuestas. Anuario de Psicología, 39(1), 59-73.

Expósito, F., Moya, M. C., \& Glick, P. (1998). Sexismo ambivalente: Medición y correlatos. Revista de Psicología Social, 13(2), 159-169. doi:http://doi.org/10.1174/021347498760350641

Ferrer, V., \& Bosch, E. (2013). Del amor romántico a la violencia de género: Para una coeducación emocional en la agenda educativa. Profesorado: Revista de Currículum y Formación del Profesorado, 17(1), 107-122.

Ferrer, V.A., Bosch, E., \& Navarro, C. (2010). Los mitos románticos en España. Boletín de Psicología (99), 7-31.

Ferrer, M. V., Bosch, E., Navarro, C., Ramis, M. C., \& García, E. (2008). El concepto de amor en España. Psicothema, 20(4), 589-595.

Flores-Kanter, P. E., Domínguez-Lara, S., Trógolo, M. A., \& Medrano, L. A. (2018). Best practices in the use of bifactor models: Conceptual grounds, fit indices and complementary indicators. Revista Evaluar, 18(3), 44-48.

doi:http://dx.doi.org/10.35670/16674545.v18.n3.22221

Flores, P., \& Browne, R. (2017). Jóvenes y patriarcado en la sociedad TIC: Una reflexión desde la violencia simbólica de género en redes sociales. Revista Latinoamericana de Ciencias Sociales, Niñez y Juventud, 15(1), pp. 147-160. doi:10.11600/1692715x.1510804082016.

García, A., \& Cedillo, P. (2011). Tras los pasos del amor: Un recuento desde las ciencias sociales. Estudios Sociológicos, 29(86), 551602.

García, J., Hernández, C. I., \& Monter, N. S. (2019). Amor romántico entre estudiantes universitarios (hombres y mujeres), una mirada desde la perspectiva de género. La Ventana, 49, 218-247.

García, N., \& Montenegro, M. (2014). Re/pensar las Producciones Narrativas como propuesta metodológica feminista. Athenea Digital, 14(4), 63-88.

doi:http://dx.doi.org/10.5565/rev/athenea.1361
Glick, P., \& Fiske, S. T. (1996). The Ambivalent Sexism Inventory: Differentiating hostile and benevolent sexism. Journal of Personality and Social Psychology, 70(3), 491-512.

doi:http://doi.org/10.1037/0022-

3514.70.3.491

Glick, P., Sakall1-Uğurlu, N., Akbaş, G., Metin, Í., \& Ceylan, S. (2016). Why do women endorse honor beliefs? Ambivalent sexism and religiosity as predictors. Sex Roles, 75, 543554.

doi:https://doi.org/10.1007/s11199-015-0550-5

Herrera, C. (2014). Amor romántico y desigualdad de género. Revista Feminista Casa de la Mujer, 20(2), 79-95.

Horn, J. L. (1965). A rationale and test for the number of factors in a factor analysis. Psychometrika, 30, 179-185. doi:https://doi.org/10.1007/BF02289447

Lagarde, M. (2001). Claves feministas para la negociación del amor. Managua: Puntos de Encuentro.

Lee, T. L., Fiske, S. T., Glick, P., \& Chen, Z. (2010). Ambivalent sexism in close relationships: (Hostile) power and (benevolent) romance shape relationship ideals. Sex Roles, 62(7-8), 583-601. doi:https://doi.org/10.1007/s11199-010-9770-x

Lloret-Segura, S., Ferreres-Traver, A., HernándezBaeza, A., \& Tomás-Marco, I. (2014). Exploratory item factor analysis: A practical guide revised and up-dated. Anales de Psicología, 30(3), 1151-1169. doi:https://doi.org/10.6018/analesps.30.3.199361

Lorenzo-Seva, U., \& Ferrando, P. J. (2006). FACTOR: A computer program to fit the exploratory factor analysis model. Behavioral Research Methods, 38(1), 88-91. doi:https://doi.org/10.3758/BF03192753

Lozano-Verduzco, I., Romero-Mendoza, M., \& Marín-Navarrete, R. (2016). Violence narratives of Mexican women treated in mutual-aid residential centers for addiction treatment. Substance Abuse Treatment, Prevention, and Policy, 11(39), 1-10. doi:https://dx.doi.org/10.1186\%2Fs13011016-0083-0

MacCallum, R. C., Widaman, K. F., Zhang, S., \& Hong, S. (1999). Sample size in factor analysis. Psychological Methods, 4(1), 84-99. 
doi:https://psycnet.apa.org/doi/10.1037/1082989X.4.1.84

Marques-Fagundes, A. L., Megías, J. L., GarcíaGarcía, D. N., \& Petkanopoulou, K. (2015). Ambivalent sexism and egalitarian ideology in perception of psychological abuse and (in)vulnerability to violence. Revista de Psicología Social, 30(1), 31-59.

doi:https://doi.org/10.1080/02134748.2014.99 1519

Medrano, L. A., \& Muñoz-Navarro, R. (2017). Aproximación conceptual y práctica a los modelos de ecuaciones estructurales. Revista Digital de Investigación en Docencia Universitaria, 11(1), 219-239. doi:http://dx.doi.org/10.19083/ridu.11.486

Montesanti, S. R., \& Thurston, W. E. (2015). Mapping the role of structural and interpersonal violence in the lives of women: implications for public health interventions and policy. BMC Women's Health, 15(100), 113. doi:https://doi.org/10.1186/s12905-015-0256-4

Montoya, O. (2007). Aplicación del análisis factorial a la investigación de mercados: Caso de estudio. Scientia et Technica, 1(35), 281286.

Nava-Reyes, M. A., Rojas-Solís, J. L., Greathouse, L. M., \& Morales, L. A. (2018). Gender roles, sexism and myths of romantic love in Mexican adolescents. Interamerican Journal of Psychology, 52(1), 102-111. doi:http://dx.doi.org/10.30849/rip/ijp.v52i1.341

Overall, N. C., \& Hammond, M. D. (2018). How intimate relationships contribute to gender inequality: Sexist attitudes encourage women to trade off career success for relationship security. Policy Insights from the Behavioral and Brain Sciences, 5(1), 40-48. doi:https://doi.org/10.1177/2372732217745096

Penagos, A., Rodríguez, M., Carrillo, S., \& Castro, J. (2006). Apego, relaciones románticas y autoconcepto en adolescentes bogotanos. Universitas Psychologica, 5(1), 21-36.

Rocha, B. L., Avendaño, C. E., Barrios, M. A., \& Polo, A. (2017). Actitudes hacia el amor en relaciones románticas de jóvenes universitarios. Praxis \& Saber, 8(16), 155178. doi:https://doi.org/10.19053/22160159.v8.n16 .2017 .4596

Rodriguez, A., Reise, S. P., \& Haviland, M. G. (2016). Evaluating bifactor models: Calculating and interpreting statistical indices. Psychological Methods, 21(2), 137150. doi:https://doi.org/10.1037/met0000045

Rodríguez-Castro, Y., Lameiras-Fernández, M., Carrera-Fernández, M. V., \& Vallejo-Medina, P. (2013). La fiabilidad y validez de la escala de mitos hacia el amor: Las creencias de los y las adolescentes. Revista de Psicología Social, 28(2), 157-168.

doi:http://dx.doi.org/10.1174/0213474138061 96708

Rodríguez, Y., Lameiras, M., \& Carrera, M. V. (2009a). Validación de la versión reducida de las escalas ASI y AMI en una muestra de estudiantes españoles. Psicogente, 12(22), 284-295.

Rodríguez, Y., Lameiras, M., Carrera, M. V., \& Faílde, J. M. (2009b). Aproximación conceptual al sexismo ambivalente: Estado de la cuestión. SUMMA Psicológica UST, 6 (2), 131-142.

Rodríguez, Y., Lameiras, M., \& Carrera, M. V. (2015). Amor y sexismo: Una peligrosa relación en los y las adolescentes gallegos/as. Revista de Estudios e Investigación en Psicología y Educación, Extr.(2), 11-14. doi:http://doi.org/10.17979/reipe.2015.0.02.234

Ruiz, M. A., Pardo, A., \& San Martín, R. (2010). Modelos de ecuaciones estructurales. Papeles del Psicólogo, 31(2), 34-45.

Sánchez-Prada, A., Delgado-Álvarez, C., BoschFiol, E., \& Ferrer-Pérez, V. A. (2019). Aportaciones sobre la Medición de Creencias acerca del Maltrato a la Mujer (IBWB) en Población Española. Revista Iberoamericana de Diagnóstico y Evaluación - e Avaliação Psicológica, 4(53) 49-62. doi:https://doi.org/10.21865/RIDEP53.4.01

Sánchez-Sicilia, A., \& Cubells, J. (2019). Discurso amoroso adolescente: Análisis del repertorio del amor romántico en el programa chicos y chicas. Athenea Digital, 19(2), 1-31. doi:https://doi.org/10.5565/rev/athenea.2429

Schäfer, G. (2008). Romantic love in heterosexual relationships: Women's experiences. Journal of Social Sciences, 16(3), 187-197. 
doi:10.1080/09718923.2008.11892617

Schreiber, J. B., Nora, A., Stage, F. K., Barlow, E. A., \& King, J. (2006) Reporting structural equation modeling and confirmatory factor analysis results: A review. The Journal of Educational Research, 99(6), 323-337. doi:https://doi.org/10.3200/JOER.99.6.323338

Smits, I. A. M., Timmerman, M. E., Barelds, D. P. H., \& Meijer, R.R. (2015). The Dutch symptom checklist-90-revised: Is the use of the subscales justified? European Journal of Psychological Assessment, 31, 263-271. doi:https://doi.org/10.1027/10155759/a000233

Stucky, B. D., \& Edelen, M. O. (2015). Using hierarchical IRT models to create unidimensional measures from multidimensional data. En S.P. Reise \& D.A. Revicki (Eds.), Handbook of item response theory modeling: Applications to typical performance assessment (pp. 183-206). New York: Routledge.

Yela, C. (2000). El amor desde la Psicología Social: Ni tan libres ni tan racionales. Madrid: Pirámide.

Yela, C. (2003). La otra cara del amor: Mitos, paradojas y problemas. Encuentros en Psicología Social, 1(2), 263-267. 


\section{Anexo I.}

Escala de Mitos del Amor Romántico (SMRL)

\begin{tabular}{|c|c|c|c|c|c|}
\hline & 1 & 2 & 3 & & 5 \\
\hline 1. El amor verdadero puede superar cualquier dificultad o problema. & & & & & \\
\hline 2. Todas las personas encontrarán en algún momento a su alma gemela. & & & & & \\
\hline 3. Cuando se encuentra a la pareja perfecta, no hay que dejarla marchar. & & & & & \\
\hline $\begin{array}{l}\text { 4. Una persona no será plenamente feliz hasta que no encuentre una pareja que le } \\
\text { complemente. }\end{array}$ & & & & & \\
\hline 5. Solo es amor verdadero cuando se mantiene la pasión de los primeros meses. & & & & & \\
\hline 6. No se puede amar a más de una persona al mismo tiempo. & & & & & \\
\hline 7. El amor verdadero debe terminar en matrimonio. & & & & & \\
\hline $\begin{array}{l}\text { 8. Una persona enamorada se preocupa más por el bienestar de su pareja que por el } \\
\text { suyo. }\end{array}$ & & & & & \\
\hline $\begin{array}{l}\text { 9. Amar a alguien es hacer todo por esa persona, aunque en ocasiones conlleve } \\
\text { hacer cosas que no te gustan. }\end{array}$ & & & & & \\
\hline 10. Los celos son una demostración de amor hacia la pareja. & & & & & \\
\hline $\begin{array}{l}\text { 11. Los gritos e insultos de la pareja se pueden perdonar siempre que haya amor } \\
\text { verdadero. }\end{array}$ & & & & & \\
\hline
\end{tabular}

Nota. 1, completamente en desacuerdo; 2, en desacuerdo; 3, ni de acuerdo ni desacuerdo; 4, de acuerdo; 5, completamente de acuerdo. 